\title{
Metodología para el diagnóstico de la red de estaciones agroclimatológicas automatizadas en el estado de Nuevo León, México
}

Automated agro-climatic stations diagnostic network methodology in the state of Nuevo Leon, Mexico

\author{
Carlos Miguel Ramos-Cruz ${ }^{1 *}$, Isidro Humberto Almeyda-León ${ }^{1}$, Lorenzo Danilo Granados-Rivera ${ }^{1}$, \\ Rogelio Molina-Muñoz ${ }^{2}$, Emilia Raquel Pérez-Evangelista ${ }^{3}$
}

\section{RESUMEN}

De las 38 estaciones meteorológicas automatizadas (EMA) que existen en Nuevo León, México, 50\% funciona de forma correcta, mientras que se desconoce las condiciones del resto de las estaciones. El presente estudio propone el diseño de una metodología para diagnosticar las EMA y determinar las causas de su mal funcionamiento. De acuerdo con los resultados obtenidos, los motivos fueron el daño de los sensores de precipitación (56.8\%), velocidad del viento (38.4\%) y dirección del viento (30.7\%). Para hacer un diagnóstico de las estaciones es necesario comprobar el correcto funcionamiento de la unidad trasmisora, paneles solares, sensores y baterías, así como verificar que su ubicación geográfica sea correcta. En conclusión, con base en la presente metodología, se determinó que las principales causas del mal funcionamiento de las EMA en el estado son la falta de supervisión y mantenimiento y poco conocimiento de la utilidad de la información que éstas generan.

PALABRAS ClAVE

predicción climática, régimen de lluvia, programación de riego

\begin{abstract}
In Nuevo León, Mexico, 50\% of the automated weather stations (AWS) is working properly, but for the rest, this data is unknown. The present study proposes a methodology to diagnose the AWS and determine the causes of malfunction. According to the results, the causes were the damage of the sensors of precipitation (56.8\%), wind speed (38.4\%) and wind direction (30.7\%). In conclusion, to make a diagnosis of the AWS, it is necessary to check the correct operation of the transmitter unit, solar panels, sensors and batteries, as well to verify the correct geographical location of the stations. In conclusion, based on the present methodology the main causes of the malfunctioning of the AWS in Nuevo León are the lack of supervision and maintenance, and the little knowledge about how useful the information is that they generate.
\end{abstract}

KEYWORDS

climate prediction, rainfall regime, irrigation schedule

\footnotetext{
${ }^{1}$ Campo Experimental General Terán, Instituto Nacional de Investigaciones Forestales, Agrícolas y Pecuarias. General Terán, Nuevo León, México.

${ }^{2}$ Instituto Tecnológico de Pabellón de Arteaga. Pabellón de Arteaga, Aguascalientes, México.

${ }^{3}$ Unidad Regional Universitaria de Zonas Áridas, Universidad Autónoma Chapingo. Bermejillo, Durango, México.

*Autor para correspondencia: Km 31 Carretera Montemorelos-China. CP 67400 General Terán, Nuevo León, México.

Correo electrónico: ramos.carlos@inifap.gob.mx
}

Recibido: 6 de junio de 2018.

Aceptado: 24 de octubre de 2018. 


\section{INTRODUCCIÓN}

La eficiencia en la producción de alimentos depende de diversos factores como variedad de semilla, el tipo de suelo, las actividades del hombre y el clima (FAO 2014). Por tal motivo, el clima es una variable clave en la producción de alimentos. Lo anterior, es cada vez más importante debido a que el avance tecnológico ha transformado la agricultura en una operación compleja y de gran escala y ello obliga a que su sustento e incremento requieran de decisiones basadas en información, entre las más importantes, la climática.

Si bien en México los tomadores de decisiones en materia de agricultura cuentan con escasa información climática para la planeación del manejo de los cultivos, se han desarrollado proyectos encaminados a resolver dicho problema (Grageda et al. 2012). Esta necesidad de contar con información meteorológica confiable ha propiciado que en las entidades federativas se establezcan redes agro-meteorológicas (Medina et al. 2008) para generar información pertinente con las necesidades de cada región (Bravo et al. 2014).

En particular, en Nuevo León se cuenta con una red agroclimática de 38 estaciones meteorológicas automatizadas (EMA), distribuidas en las principales zonas agrícolas del estado y administradas por el Instituto Nacional de Investigaciones Forestales, Agrícolas y Pecuarias (INIFAP) y Fundación Produce Nuevo León. El registro de las variables climáticas de dicha red se contabiliza cada 15 minutos y los datos son enviados al Laboratorio Nacional de Modelajes y Sensores Remotos (LNMySR) del INIFAP ubicado en el Campo Experimental Pabellón, Aguascalientes (Martínez et al. 2008; Mariles et al. 2013). Esta información y los registros históricos pueden ser consultados en tiempo real de forma gratuita en el portal http://clima.inifap.gob.mx.

Es importante mencionar que, antes de ser puestos a disposición de los usuarios para su consulta y descarga, los datos son sometidos a pruebas de control de calidad para identificar y eliminar información errónea (Rodríguez y Ramos 2017). Las variables registradas por las EMA son las de mayor importancia para caracterizar los procesos de intercambio entre al área circundante de la hoja y el medio ambiente. El análisis conjunto de datos de temperatura, humedad relativa y precipitación pluvial, dirección y velocidad del viento y radiación solar, en especies cultivadas (anuales y perennes) permite generar programas de control de plagas y enfermedades y polinización de los cultivos. Dependiendo de la existencia de bases

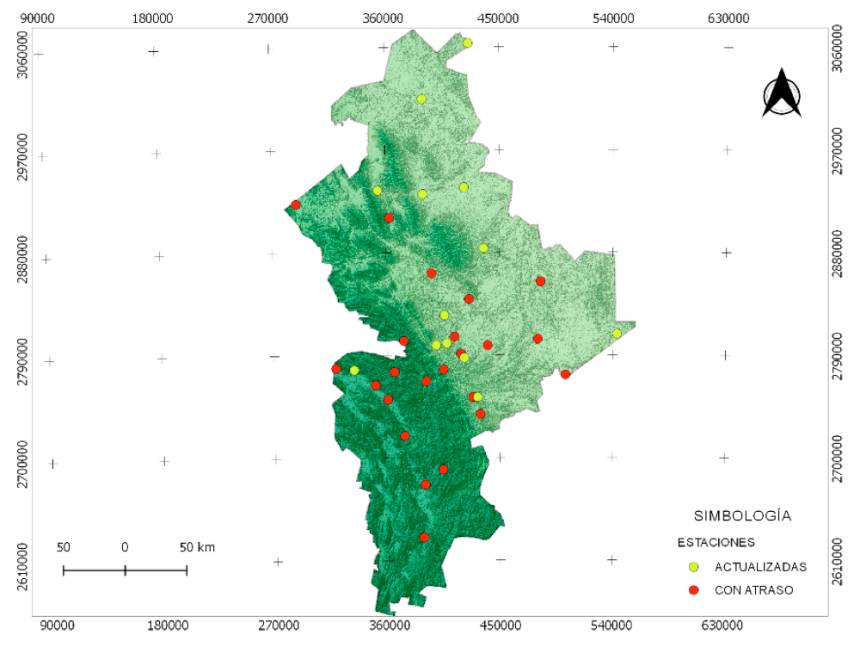

Figura 1. Distribución geográfica de la red de estaciones climatológicas del estado de Nuevo León, México.

de datos del clima se pueden alimentar modelos de pronóstico para cosecha de los cultivos a corto plazo y estudios de cambio climático (Rodríguez y Ramos 2017).

No obstante, su utilización en estos momentos es limitada debido a que de las 38 EMA que existen en Nuevo León, sólo 13 se encuentran operando en línea. Debido a esto, el objetivo de este estudio es generar una metodología o técnica para eficientizar la operación de las EMA de la red de estaciones agroclimatológicas automatizadas en Nuevo León.

\section{Materiales Y MÉtodos}

El estudio se realizó en el estado de Nuevo León, México, el cual cuenta con una red de 38 EMA administradas por el INIFAP y la Fundación Produce Nuevo León (figura 1). Cada estación cuenta con sensores para medir temperatura del aire $(\mathrm{T})$, humedad relativa $(\mathrm{HR})$, precipitación $(\mathrm{P})$, dirección del viento $(\mathrm{DV})$, velocidad del viento $(\mathrm{VV})$ y radiación solar (Rad).

Con la finalidad de realizar un diagnóstico de cada una de las EMA, se realizó un recorrido y se evaluaron los distintos componentes que las integran, de la siguiente manera:

Diagnóstico de sensores y paneles. Se desmontó la estación de la base y se extrajo la unidad transmisora de radio (UTR), posteriormente, se desconectaron los cables de los sensores y del panel. Después, se comprobó el funcionamiento de cada sensor con un tester sensores-paneles (marca ADCOM Telemetry ${ }^{\circledR}$ modelo A511) y se realizó un reporte de cada EMA analizada. 
Cuadro 1. Estaciones climatológicas diagnosticadas de la red estatal de Nuevo León, México.

\begin{tabular}{|c|c|c|c|c|c|c|c|c|c|c|}
\hline \multirow{2}{*}{ Nombre EMA } & \multirow{2}{*}{$\begin{array}{c}\text { Días } \\
\text { Atraso }\end{array}$} & \multirow{2}{*}{ RAdio } & \multirow{2}{*}{ Panel } & \multirow{2}{*}{ Batería } & \multicolumn{6}{|c|}{ Sensores } \\
\hline & & & & & $\mathrm{T}$ & HR & DV & VV & RAD & $\mathbf{P}$ \\
\hline Aramberri & 800 & 0 & 0 & 0 & 0 & 0 & 1 & 1 & 0 & 0 \\
\hline Los Rayones & 800 & 0 & 0 & 0 & 0 & 0 & 1 & 1 & 0 & 0 \\
\hline San José de Raíces & 2202 & EP & EP & EP & EP & EP & EP & EP & EP & $\mathrm{EP}$ \\
\hline INIFAP & 800 & 0 & 1 & 0 & 0 & 0 & 0 & 0 & 0 & 0 \\
\hline Vivero el Llano & 1020 & 0 & 0 & 0 & 0 & 0 & 0 & 1 & 0 & 1 \\
\hline El Naranjo & 750 & 1 & 0 & 1 & 0 & 0 & 0 & 0 & 0 & 0 \\
\hline Cerro de Agua & 253 & $\mathrm{EP}$ & EP & EP & EP & EP & $\mathrm{EP}$ & EP & EP & EP \\
\hline El Berrendo & 800 & 0 & 1 & 0 & 0 & 0 & 0 & 0 & 0 & 0 \\
\hline Rancho María Josefina & 359 & 0 & 0 & 1 & 1 & 1 & 0 & 1 & 0 & 1 \\
\hline $\begin{array}{l}\text { Facultad de Agronomía } \\
\text { UANL }\end{array}$ & 800 & 0 & 1 & 0 & 0 & 0 & 0 & 0 & 0 & 1 \\
\hline San Isidro & 1020 & EP & EP & 0 & EP & $\mathrm{EP}$ & $\mathrm{EP}$ & EP & EP & EP \\
\hline Sandia & 800 & 0 & 1 & 0 & 0 & 0 & 0 & 0 & 0 & 1 \\
\hline La Ascensión & 349 & 0 & 1 & 0 & 0 & 0 & 0 & 0 & 0 & 1 \\
\hline Dr. Arroyo & 768 & EP & EP & EP & EP & EP & $\mathrm{EP}$ & EP & EP & EP \\
\hline San Gabriel & 2215 & EP & EP & EP & EP & EP & EP & EP & EP & EP \\
\hline Lab. Biotecnología & 264 & 1 & 0 & 0 & 0 & 0 & 0 & 0 & 0 & 1 \\
\hline San Rafael & 710 & 0 & 0 & 0 & 0 & 0 & 0 & 0 & 0 & 0 \\
\hline La Leona & 1781 & EP & EP & EP & EP & $\mathrm{EP}$ & $\mathrm{EP}$ & EP & EP & EP \\
\hline Las Milpillas & 2238 & 0 & & 1 & 1 & 1 & 1 & 1 & 1 & 1 \\
\hline Total & & 2 & 5 & 3 & 2 & 2 & 4 & 5 & 1 & 7 \\
\hline
\end{tabular}

EMA: estación meteorológica automatizada, EP: estación perdida, T: temperatura del aire, HR: humedad relativa, DV: dirección del viento, VV: velocidad del viento, Rad: radicación solar, P: precipitación; $0=$ sensor en buen estado, $1=$ sensor dañado

Funcionamiento de la unidad transmisora de datos. Para verificar su funcionamiento, así como la correcta configuración de la IP para envío de la información al LNMySR se utilizó un cable programador para UTR USB-5pin y el software ADCOM Telemetry UTR Configurator.

Inspección de baterías. Para comprobar que las baterías no presentaran daño, se abrió la UTR y desconectó el paquete de baterías de la placa electrónica por medio del conector PCB. El estado de carga se midió con un multímetro digital.

Verificación de la ubicación. Debido a que algunos datos registrados en el LNMySR, respecto a la ubicación de las EMA, no coincidían con las coordenadas reales de las mismas, se verificó dicha información, y se hicieron las correcciones pertinentes. Para esto se utilizó un GPS (marca GARMIN ${ }^{\circledR}$ modelo Etrex 20).

\section{RESULTADOS Y DISCUSIÓN}

En la red de estaciones meteorológicas del estado de Nuevo León se encontraron 15 EMA con un atraso en el envío de información a la base de datos del LNMySR menor a 3 años, y 4 con atraso mayor a 6 años. Asimismo, se encontró que $38.4 \%$ de los paneles estaban dañados, lo cual ocasionó que las baterías dejaran de cargar y en consecuencia las unidades transmisoras de datos no enviaran información a la base A850 Telemetry Gateway.

Los sensores que presentaron daño mayor fueron los de precipitación (53.8\%), velocidad del viento (38.4\%) y dirección del viento (30.7\%) (cuadro 1). En particular, en el sensor de precipitación la principal causa de daño fueron los cables desoldados del contador electrónico a la UTR; asimismo, colectores 
de precipitación quebrados, tapados por tierra, hojas de árboles e insectos (telarañas y panales de avispas), y ello impedía que las lecturas se realicen de manera correcta. Lo anterior, es debido a la falta de mantenimiento (Velasco et al. 2017), el cual se recomienda realizarse cada seis meses en estaciones cercanas a poblaciones y cada año en estaciones de difícil acceso (WMO 2018b). Estos mantenimientos se pueden diferenciar en dos tipos: preventivo y correctivo. El primero consiste en una inspección de la EMA para prevenir averías y prolongar el tiempo de vida y funcionamiento de los componentes, así como el mantenimiento de las instalaciones. El mantenimiento correctivo, consiste en la reparación, ajuste o sustitución de los elementos que ya no funcionen de forma adecuada (AEMeT 2016). Realizar mantenimientos periódicos garantizan mayor calidad y homogeneidad de los datos climáticos (WMO 2018b).

Las 6 estaciones ubicadas en San José de Raíces, Cerro de agua, San Isidro, Dr. Arroyo, San Gabriel y La Leona ya no se encontraron instaladas en su ubicación. De acuerdo con la información proporcionada por los encargados de los predios, estas fueron desinstaladas $\mathrm{y}$ bandalizadas por personas ajenas al personal encargado de su resguardo. Asimismo, durante el diagnostico se repararon tres, las cuales presentaron problemas en la configuración de IP (Aramberri, Vivero el Llano) y antena desconectada (San Rafael), lo cual facilitó su reparación y ahora buen funcionamiento.

Las EMA que presentaron problemas de ubicación (INIFAP, San Isidro y San Rafael) tenían un error mayor a $500 \mathrm{~m}$, lo cual podría afectar los pronósticos generados con su información. Por lo tanto, se realizó el ajuste de la ubicación en el portal de la red nacional de estaciones.

En Nuevo León, $65.8 \%$ de las EMA no se encuentran en condiciones operables, de forma particular las ubicadas en la región centro y sur; por lo tanto, más de $60 \%$ de la superficie estatal se encuentra sin información climática. Esto es preocupante debido a que en dichas regiones se desarrolla $82 \%$ de la agricultura (SAGARPA 2015), y generan 1.5\% de los productos agrícolas cosechados en el país, con valor de producción de \$3 032 millones de pesos (Pantoja y Flores 2018).

La información que proporciona la Red Nacional de Estaciones Agroclimáticas Automatizadas (RNEAA), administrada por el INIFAP, es la única a nivel nacional cuyo enfoque es totalmente dirigido a auxiliar al sector agrícola (Velasco et al. 2017). Las variables registradas por las EMA son las más importantes para caracterizar los procesos de intercambio entre el medio y la atmósfera (Rodríguez y Ramos 2017). Además, el INIFAP ha sido declarado por la SAGARPA como autoridad técnica competente para realizar dictámenes de ocurrencia de desastres naturales, que se realizan por medio de los datos registrados por la RNEAA y la interpretación agronómica de la afectación ocurrida por algún evento meteorológico extremo (INIFAP 2018). Disponer de información climática facilita la toma de decisiones de forma inteligente, al permitir una mejor gestión de los riesgos y oportunidades en los sectores productivos sensibles a la variación del clima (WMO 2018a), como lo es la agricultura (WMO 2016), sector que pierde grandes volúmenes de producción por efectos climáticos (Hay 2007).

Para resolver esta situación, es necesario contar con datos climáticos históricos, en tiempo real y confiables, los cuales sirven, entre otras cosas, para realizar el combate a plagas que causan enfermedad en los cultivos, el diseño de la programación de siembra, planeación de la aplicación de agroquímicos, momentos adecuados de la cosecha y el cálculo del balance hídrico (Rodríguez y Ramos 2017), este último, de forma general, ayuda a hacer un uso eficiente del agua para riego (Hernández 1993). Al respecto, la WMO (1984) menciona que a través del cálculo del balance hídrico se puede hacer eficiente el uso del agua en la agricultura en $90 \%$. Por su parte, Sifuentes et al. (2016) calcularon el requerimiento hídrico del cultivo de papa a través de información obtenida con las EMA y, reportaron que es posible incrementar la eficiencia del agua en $80 \%$ en riego por gravedad, $90 \%$ en riego por aspersión y $95 \%$ en riego por goteo. De la misma forma, Delgado et al. (2018) reportaron que al realizar una programación del riego con base en datos climáticos de las EMA para el cultivo de naranja, se puede obtener un ahorro de $13 \%$ en el consumo de energía eléctrica, con lo cual se garantiza una disminución en los costos de producción y por ende se incrementa la rentabilidad del sistema. Los estudios anteriores, ponen de manifiesto la importancia de los datos meteorológicos generados por la EMA en la agricultura y de forma particular, en el uso eficiente del agua, que en la actualidad y más aún en el futuro, es un tema de alta relevancia a nivel mundial. Sin embargo, existe poca o ninguna información retroalimentaria del sector agrícola sobre cómo utilizar los datos climáticos en la toma de decisiones, por lo que se requiere realizar más capacitación sobre el uso de dicha información por parte de los usuarios finales (WMO 2018c). 


\section{CONCLUSIONES}

En la búsqueda de una metodología o técnica que permita realizar un diagnóstico de las EMA en Nuevo León y en el país, se desarrolló una metodología que consiste en verificar el funcionamiento de sensores y paneles solares, unidad transmisora de datos, baterías y ubicación. Con base en esta metodología, el diagnostico que se hizo de la red de estaciones meteorologías de Nuevo León determinó que las principales causas de su mal funcionamiento, se debe a la falta de mantenimiento, asimismo, el poco interés del resguardo, por parte de los propietarios de los predios donde se encuentras instaladas. Lo anterior, es posible que se deba al desconocimiento de la utilidad de la información que generan estas estaciones. Por tanto, se recomienda realizar un adecuado mantenimiento de la red climática del estado, así como campañas de concientización y capacitación respecto a la importancia y utilidad de la información climática en la agricultura para propiciar el cuidado de dichos instrumentos. 


\section{LITERATURA CITADA}

Agencia Estatal de Meteorología. [internet]. 2016. Mantenimiento de estaciones meteorológicas automáticas. [cited 2018 may]. Disponible en: https://aemetblog. es/2016/05/10/mantenimiento-de-estaciones-meteorologicas-automaticas/

Bravo E, Cano M, López P, Mariles L, González M, González L. 2014. Aplicaciones de la Red de estaciones agroclimatológicas automatizadas del estado de Oaxaca. Instituto Nacional de Investigaciones Forestales, Agrícolas y Pecuarias. Campo Experimental Valles Centrales de Oaxaca. Etla, México.

Delgado G, Ramos CM, Miguel E. 2018. Indicadores de eficiencia agronómica en sistemas de riego de baja presión en la región citrícola de Nuevo León. Memoria de la XXX Semana Internacional de Agronomía FAZ-UJED. Gomez Palacio, México.

[FAO] Organización de las Naciones Unidas para la Alimentación y la Agricultura. 2014. Agricultura familiar en América Latina y el Caribe: Recomendaciones de política. Santiago, Chile.

Grageda J, Jiménez A, Rodríguez J, Fu A, Pérez B, Borrón R. 2012. Uso de la información de una red de estaciones meteorológicas automatizadas en Sonora. Revista de Ciencias Biológicas y de la Salud 14(1): 13-17.

Hay J. 2007. Extreme weather and climate events, and farming risks. In: Sivakumar MVK, Motha RP, editors. Managing weather and climate risks in agriculture. Berlin, Springer Berlin Heidelberg. 1-19.

Hernández ML. 1993. La agroclimatologia, intrumento de la planificación agricola. Geographicalia 30: 213-228.

Instituto Nacional de Investigaciones Forestales, Agrícolas y Pecuarias. 2018. Laboratorio Nacional de Modelaje y Sensores Remotos. INIFAP - Campo Experimental Pabellón Km. 32.5 Carretera Panamericana Aguascalientes-Zacatecas Pabellón de Arteaga, Aguascalientes, México. [cited 2018 september]. Disponible en: http:// clima.inifap.gob.mx/lnmysr/Principal/Index

Mariles V, Cano M, Bravo E, López P, Maciel L, González M, González L. 2013. Red de estaciones climatológicas automatizadas en el estado de Oaxaca. Instituto Nacional de Investigaciones Forestales, Agrícolasy Pecuarias. Campo Experimental Valles Centrales de Oaxaca. Santo Domingo Barrio Bajo, México.

Martínez O, Cano A, Berlanga C. 2008. La medición del clima y sus aplicaciones en las actividades agricolas del estado de Coahuila. Instituto Nacional de Investigaciones Forestales, Agricolas y Pecuarias. Campo Experimental Saltillo. Saltillo, México.

Medina G, Grageda J, Ruiz J, Báez A. 2008. Uso de estaciones meteorológicas en la agricultura. Instituto Nacional de Investigaciones Forestales, Agrícolas y Pecuarias. Campo Experimental Zacatecas. Folleto Informativo Núm. 50. Calera, México.

Pantoja GM, Flores F. 2018. El sector citrícola de Nuevo León: caracterización del sistema agroalimentario como plataforma de integración del productor con la agroindustria. Región y Sociedad 30(71): 1-46. https:// doi.org/10.22198/rys.2018.71.a385

Rodríguez V, Ramos F. [internet]. 2017. Agrometeorología: Del monitoreo del clima a la agricultura climicamente inteligente. Hortalizas, México. [cited 2018 may]. Disponible en: http://www.hortalizas.com/nutricion-vegetal/62390/

Secretaría de Agricultura, Ganadería, Desarrollo Rural, Pesca y Alimentación. 2015. Anuario Estadístico agroalimentario 2015. Monterrey, México.

Sifuentes E, Macías J, Ojeda W, González V, Salinas D, Quintana J. 2016. Gestión del riego enfocada a variabilidad climática en el cultivo de papa: aplicación al Distrito de Riego 075, Río Fuerte, Sinaloa, México. Tecnología y Ciencias del Agua 7(2): 149-168.

Velasco VD, Flores FG, González G, Flores MJ, Moreno HA. 2017. Desarrollo y validación de una estación meteorológica automatizada de bajo costo dirigida a agricultura. Revista Mexicana de Ciencias Agrícolas 6(6): 1253-1264.

World Meteorological Organization. 1984. Informe anual de la Organización Meteorologíca Mundial OMM-No. 644. Ginebra, Suiza.

World Meteorological Organization. 2016. Climate services for supporting climate change adaptation. Supplement to the technical guidelines for the national adaptation plan process WMO-No. 1170. Geneva, Switzerland.

World Meteorological Organization. 2018a. Directrices detalladas para la creación de un marco nacional para los servicios climáticos. Organización Meteorológica Mundial OMM-No. 1206. Genève, Suiza.

World Meteorological Organization. 2018b. Guidelines on Quality Management in Climate Services. World Meteorological Organization WMO-No. 1221. Geneva, Switzerland.

World Meteorological Organization. 2018c. Report on responses to the questionnaire on climate services for agriculture in regional association V (South-West Pacific). Commission for Agricultural Meteorology. World Meteorological Organization CAgM-Report No. 105. Geneva, Switzerland. 